\title{
Fluorescence-Based Rapid Detection of Microbiological Contaminants in Water Samples
}

\author{
Hervé Meder, Anne Baumstummler, Renaud Chollet, Sophie Barrier, Monika Kukuczka, \\ Frédéric Olivieri, Esther Welterlin, Vincent Beguin, and Sébastien Ribault
}

\begin{abstract}
Merck Millipore, Lab Solutions, BioMonitoring, Research \& Development, Applications Group, 39, Route industrielle de la Hardt, 67120 Molsheim, France
\end{abstract}

Correspondence should be addressed to Hervé Meder, herve.meder@merckgroup.com

Received 8 December 2011; Accepted 25 December 2011

Academic Editors: A. P. Hudson and A. L. MacHado

Copyright () 2012 Hervé Meder et al. This is an open access article distributed under the Creative Commons Attribution License, which permits unrestricted use, distribution, and reproduction in any medium, provided the original work is properly cited.

\begin{abstract}
Microbiological contamination of process waters is a current issue for pharmaceutical industries. Traditional methods require several days to obtain results; therefore, rapid microbiological methods are widely requested to shorten time-to-result. Milliflex Quantum was developed for the rapid detection and enumeration of microorganisms in filterable samples. It combines membrane filtration to universal fluorescent staining of viable microorganisms. This new alternative method was validated using European and United States Pharmacopeia definitions, with sterile water and/or sterile water artificially contaminated with microorganisms. The Milliflex Quantum method was demonstrated to be reliable, robust, specific, accurate, and linear over the whole range of assays following these guidelines. The Milliflex Quantum system was challenged to detect natural contaminants in different types of pharmaceutical purified process waters. Milliflex Quantum was demonstrated to detect accurately contaminants 3- to 7-fold faster than traditional membrane filtration method. The staining procedure is nondestructive allowing downstream identification following a positive result. The Milliflex Quantum offers a fast, sensitive, and robust alternative to the compendial membrane filtration method.
\end{abstract}

\section{Introduction}

Microbiological contamination of process waters is a key issue for pharmaceutical, biotechnology, and food and beverage industries. Traditional methods are the reference for the control of microbiological quality of water as they are reliable, easy to use and allow microorganisms identification. Nevertheless, these methods are time-consuming and laborintensive. Moreover, they depend on the ability of microorganisms to yield visible colonies after an incubation period of typically 3 days that can go up to 14 days (European and United States Pharmacopeia). This long time-to-result is a concern for industries as improvement in processes and products requires faster methods to control microbiological quality. Therefore, over the past 25 years, many technologies have been developed to reduce the time-to-result. These new alternative rapid methods have to be sensitive, accurate, and cost-effective. The most studied and used technologies are polymerase chain reaction (PCR), impedimetry, bioluminescence, enzyme-linked immunosorbent assay (ELISA), flow cytometry (FCM), and solid-phase cytometry (SPC) [1-3].

The Milliflex Quantum (Millipore, Molsheim, France) is a new system developed for the rapid detection and enumeration of microorganisms in filterable samples. It combines membrane filtration and fluorescent staining, which are two proven and widely used technologies. The detection is based on a universal enzymatic fluorescent staining of viable microorganisms. The staining procedure is nondestructive allowing downstream identification following a positive result.

This paper describes the validation of the performances of the Milliflex Quantum method. The study was done according to definitions for the validation of a quantitative estimation of viable microorganisms in a sample from European (chapter 5.1.6.) and United States (chapter $<1223>$ ) Pharmacopeia. Robustness, ruggedness, accuracy, linearity, 
range, limit of quantification, limit of detection, and specificity of the method were assessed with sterile water and/or sterile water artificially spiked with microorganisms. The Milliflex Quantum method was also challenged to detect natural contaminants in different types of pharmaceutical process waters. All results obtained with Milliflex Quantum method were compared to the traditional membrane filtration method.

\section{Materials and Methods}

2.1. Media. Prefilled Tryptic Soy Agar plates (TSA, Millipore), R2A plates (Millipore), and Sabouraud Dextrose Agar plates (SDA, Millipore) were used to promote growth of microorganisms.

2.2. Microorganisms Strains. The following American-Type Culture Collection (ATCC) strains were used to validate the Milliflex Quantum method: Candida albicans ATCC 10231, Aspergillus brasiliensis ATCC 16404, Staphylococcus epidermidis ATCC 12228, Ralstonia pickettii ATCC 27511, Brevundimonas diminuta ATCC 19146, Staphylococcus aureus ATCC 6538, Pseudomonas aeruginosa ATCC 9027, Bacillus subtilis ATCC 6633, and Escherichia coli ATCC 8739. An environmental isolate of Caulobacter sp. was also tested with Milliflex Quantum. The cultures were maintained at $-80^{\circ} \mathrm{C}$ in Tryptic Soy Broth (TSB; BioMérieux, Craponne, France) with 5\% (v/v) glycerol (Sigma-Aldrich, St. Quentin Fallavier, France) in $0.5 \mathrm{mmol} \mathrm{L}^{-1}$ HEPES buffer (Sigma-Aldrich).

2.3. Milliflex Quantum Method. The procedure used is as described by Baumstummler et al. [4]. Briefly, samples were filtered through mixed cellulose ester membranes and membranes were placed onto media agar plates and incubated at temperatures recommended by Pharmacopeia or at optimal growth temperatures. After incubation, membranes were stained for $30 \mathrm{~min}$ and placed in the Milliflex Quantum reader for counting fluorescent microcolonies. Membranes were reincubated onto media agar plates for visual counting of colony-forming unit (CFU), viability assessment, and contaminants identification. The compendial method was performed in parallel.

Fluorescence counts and CFU counts obtained after reincubation were compared to the compendial method. The fluorescence recovery and viability recovery were calculated as follows:

Fluorescence recovery (\%)

$$
=\frac{\text { Fluorescence count }}{\text { Compendial method count }} \times 100,
$$

Viability recovery (\%)

$$
=\frac{\text { CFU count after reincubation }}{\text { Compendial method count }} \times 100 \text {. }
$$

Acceptance criterion for these parameters was set to equal to or higher than $70 \%$ (European Pharmacopeia chapter 5.1.6. and United States Pharmacopeia chapter <1223>).

\subsection{Milliflex Quantum Method Validation}

2.4.1. Statistical Analysis. An Anderson-Darling test or a Goodness-Of-Fit Chi2-test was used to determine if data obtained with the Milliflex Quantum method and with the compendial method follow a normal distribution. When data are normally distributed (Anderson-Darling $P$ value $\geq$ 0.1 ; Chi2-value $\leq 4.61$ for a 5 classes distribution; Chi2-value $\leq 2.71$ for a 4 classes distribution), a one-way analysis of variance (ANOVA), a Student's two samples $t$-test or a Chi2test was performed to compare results obtained with both methods. All statistical analysis were carried out using the Minitab Statistical Software (version 14; Minitab Inc., State College, PA, USA) except the Goodness-Of-Fit Chi2-test which was performed with Microsoft Office Excel (version 2003; Microsoft, Redmond, WA, USA).

2.4.2. Negative Controls. Negative controls were carried out in parallel of microorganisms testing. One hundred $\mathrm{mL}$ of $0.9 \% \mathrm{NaCl}$ water (B. Braun Medical, Boulogne Billancourt, France) was filtered, incubated, and analyzed in the same conditions as samples containing microorganisms.

2.4.3. Incubation Time Robustness. Spiked samples were filtered, and membranes were incubated during various times before being stained following the Milliflex Quantum protocol as described previously. Membranes were reincubated to assess viability. Incubation conditions used were those required for the microbiological examination of nonsterile products in chapters of European (2.6.12. and 2.6.13.) and United States $(<61>$ and $<62>$ ) Pharmacopeia. Fluorescence and viability recoveries were determined in comparison with the compendial method. An ANOVA was used to assess the time range for stable enumeration with Milliflex Quantum.

2.4.4. Ruggedness. The effect of using different media lots, membrane lots, reagent lots, analysts, and instruments was assessed. Candida albicans and Ralstonia pickettii, spiked separately in sterile water, were detected using 2 different lots of either membranes or reagents. Moreover, ruggedness of media was evaluated on TSA with Bacillus subtilis and Escherichia coli, on SDA with Candida albicans and on R2A with Ralstonia pickettii. For each ruggedness test, two different test runs were performed. Each run was tested by a different analyst, with a different set of instruments. Fluorescence and viability recoveries were calculated, and ANOVA was performed to check if recoveries corresponding to the different tested conditions were not statistically different $(P$ value $\geq 0.05)$.

2.4.5. Accuracy, Linearity, Range, and Limit of Quantification. For each challenged microorganism spiked in sterile water, tests were performed at the following targeted spike levels per sample: $0 \mathrm{CFU}, 5 \mathrm{CFU}, 25 \mathrm{CFU}, 50 \mathrm{CFU}, 75 \mathrm{CFU}$, and 100 CFU. Milliflex Quantum method and traditional method were performed at the same time. Fluorescence and viability recoveries were calculated and a Student's two samples $t$-test was performed to check if the Milliflex Quantum counts were 
not statistically different from the traditional Milliflex counts $(P$ value $\geq 0.05)$.

Linearity, range, and limit of quantification were established from the data generated during the accuracy test. Acceptance criteria for linearity include an $R^{2}$-value greater than 0.95 (United States Pharmacopeia) and a linear regression slope between 0.8 and 1.2.

2.4.6. Limit of Detection. The microorganisms used for accuracy testing were adjusted separately to approximately $3-5 \mathrm{CFU}$ per $100 \mathrm{~mL}$ until at least $50 \%$ of the samples showed growth in the compendial method. Twenty replicate samples were assessed with each microorganism with each method. As the aim of the test was to demonstrate that the Milliflex Quantum method enabled to detect $1 \mathrm{CFU}$, the method had to detect at least one time $1 \mathrm{CFU}$ during the experiment. Furthermore, a Student's two samples $t$-test was performed to check if the Milliflex Quantum counts were not statistically different from the traditional Milliflex counts $(P$ value $\geq 0.05$ ). Finally, the equivalence between the Milliflex Quantum proportion of growth and the traditional method proportion was assessed with a Chi2-test $(P$ value $\geq 0.05)$.

2.4.7. Specificity. The specificity of Milliflex Quantum method was established from the data generated during the robustness and accuracy tests, where a panel of microorganisms was tested.

2.5. Detection of Microorganisms in Pharmaceutical Process Waters. In-process nonsterile water samples were taken at different steps of the water treatment process in 5 pharmaceutical plants. These various types of purified waters were diluted in $100 \mathrm{~mL}$ of $0.9 \% \mathrm{NaCl}$ water (B. Braun Medical) and filtered. Membranes were placed onto R2A plates (Millipore) and incubated at $32.5^{\circ} \mathrm{C}$ (European Pharmacopeia General Monographs: Water For Injections; Water Highly Purified; Water Purified). Several incubation times were tested to assess the minimal incubation time required for the fluorescence detection. After incubation, membranes were stained following the Milliflex Quantum protocol. Membranes were reincubated onto R2A plates (Millipore) for visual counting of CFU and contaminants identification. The compendial method was performed in parallel. Fluorescence and viability recoveries were determined in comparison with the compendial method.

\section{Results}

\subsection{Milliflex Quantum Method Validation}

3.1.1. Incubation Time Robustness. The robust incubation time range required for detection with Milliflex Quantum was evaluated on 10 microorganisms. Table 1 summarizes results obtained. Conforming detection of microorganisms was achieved after $22 \mathrm{~h}$ of incubation for Candida albicans and after $28 \mathrm{~h}$ for Aspergillus brasiliensis. The minimal incubation time to detect Escherichia coli and Bacillus subtilis was $8 \mathrm{~h}$ and $9 \mathrm{~h}$, respectively. The environmental isolate of

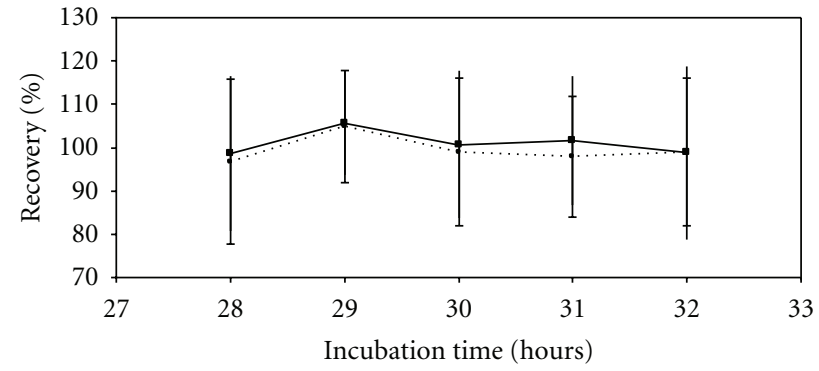

FIgURE 1: Determination of the robust incubation time range required to detect Aspergillus brasiliensis with the Milliflex Quantum method $(n=10)$. Fluorescence recovery $(\boldsymbol{\square}$, solid line); viability recovery $(\bullet$, dashed line). Standard deviation is denoted by the vertical bars (delineated with-for viability standard deviation).

Caulobacter $s p$. was detected after 28 hours. The other 5 bacteria tested needed between $12 \mathrm{~h}$ and $22 \mathrm{~h}$ of incubation. The Milliflex Quantum method was demonstrated to be robust over several incubation times for each strain (fluorescence and viability recoveries $\geq 70 \%$ and ANOVA $P$ value $\geq 0.05$, $n=10$ ). These incubation time ranges were used during the further tests of the method validation. Recoveries results over the whole tested incubation time range obtained with Aspergillus brasiliensis are presented as example in Figure 1, which proves the results stability and method robustness over the 4-hour tested range.

3.1.2. Ruggedness. Using different media lots, membrane lots and reagent lots has no significant effect on Milliflex Quantum results as ANOVA results proved that fluorescence and viability recoveries were statistically equivalent in all tested conditions (data not shown). Reproducibility of tests results is guaranteed as well with different operators and equipment sets. Therefore, the method's performance is ensured in terms of ruggedness.

3.1.3. Accuracy, Linearity, Range, and Limit of Quantification. Candida albicans was accurately detected with the method at each tested contamination level (Table 2) as fluorescence recoveries ranged from $98 \%$ to $102 \%$ and viability recoveries from $97 \%$ to $102 \%$. Moreover, no statistical differences between Milliflex Quantum and traditional method (Student's two samples $t$-test; $P \geq 0.05$ ) were found. Similar results were obtained with Aspergillus brasiliensis, Escherichia coli, and Bacillus subtilis (data not shown).

Linearity, range, and limit of quantification results obtained with microorganisms tested are showed in Table 3. A linear correlation between either fluorescence counts or viability counts and counts obtained with the compendial method were demonstrated since $R^{2}$-values varied from 0.95 to 0.98 . The detection observed with Milliflex Quantum ranged from $0 \mathrm{CFU}$ with each microorganism to 97 to $163 \mathrm{CFU}$, depending on the microorganism tested.

Limits of quantification of the Milliflex Quantum method were demonstrated to be between 4 and 10 CFU, depending on the microorganism tested. These levels are equal 


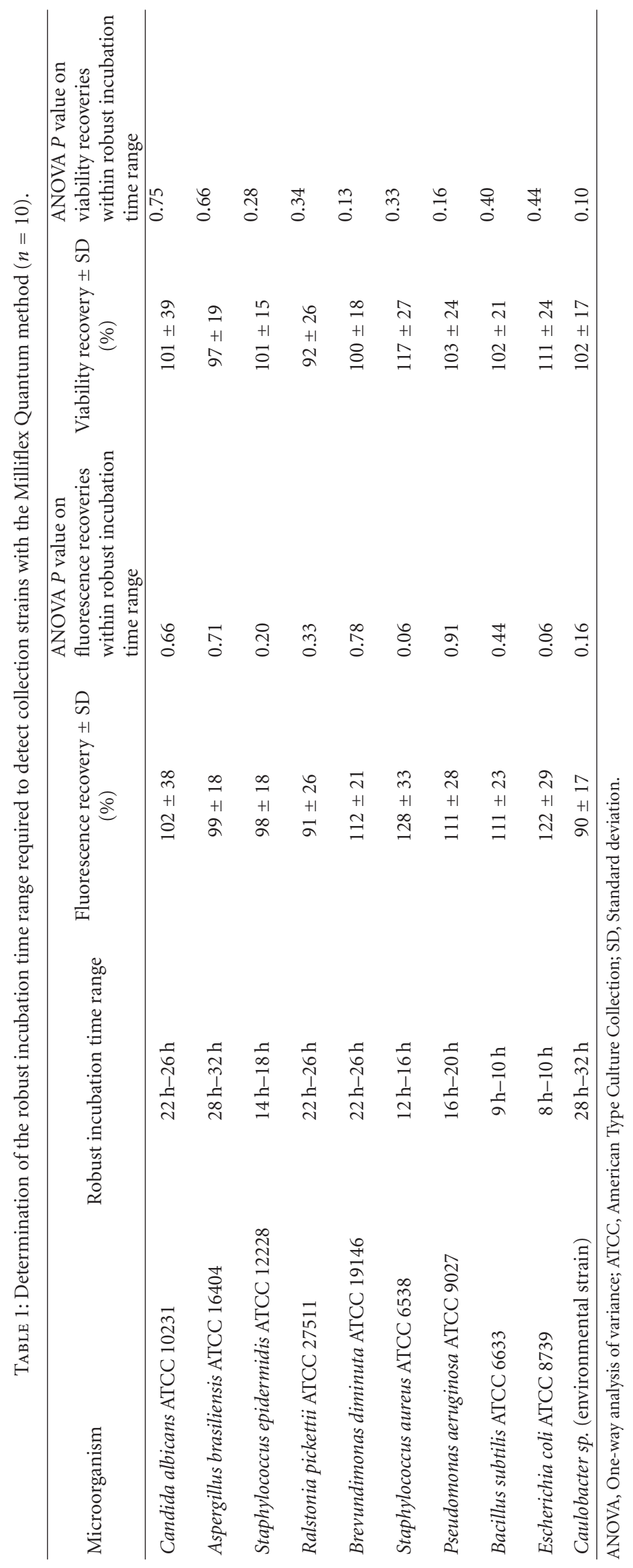




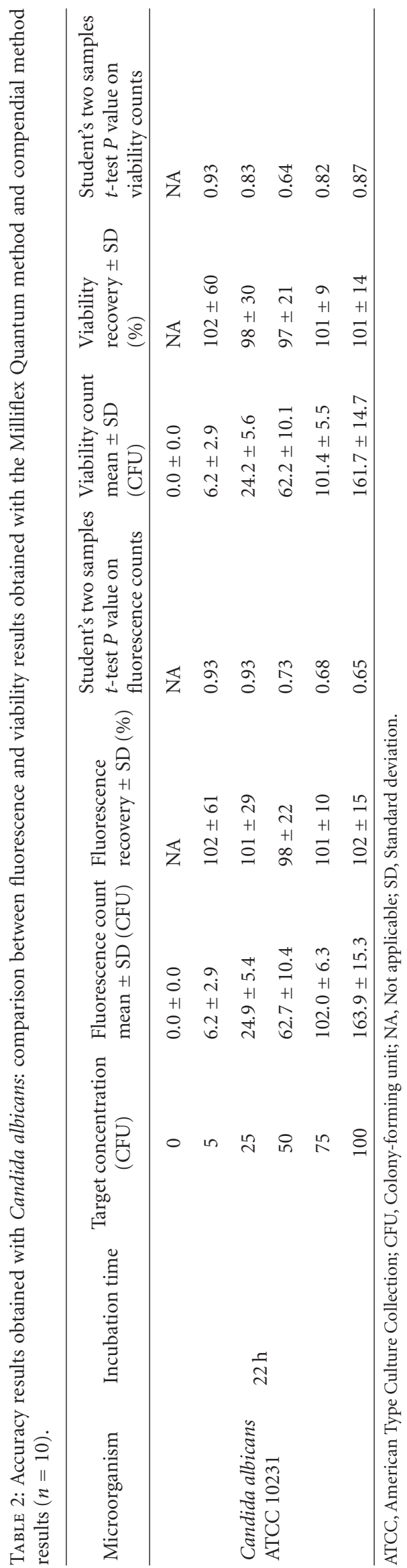




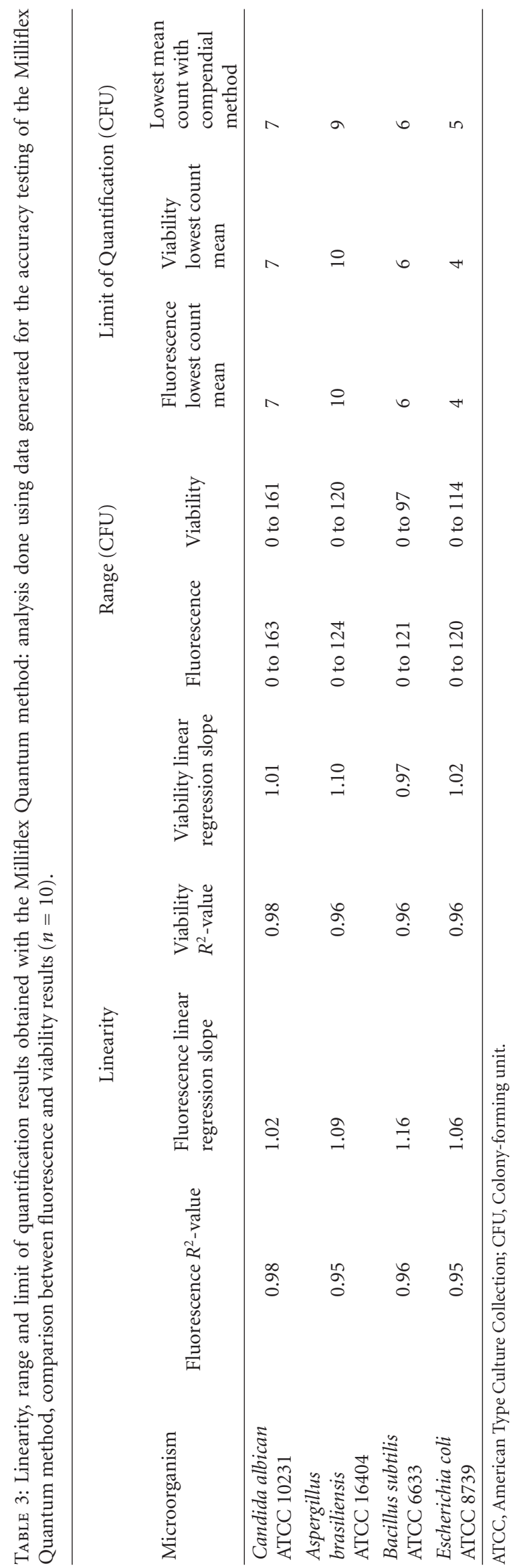


to or lower than the corresponding limit of quantification of the compendial method except with Aspergillus brasiliensis. With this strain, the limit of quantification of Milliflex Quantum method was found to be $10 \mathrm{CFU}$, while, $9 \mathrm{CFU}$ was the level demonstrated with compendial method. This latter result is explained by the Aspergillus morphology. Due to filaments and colonies merging, the colonies are more difficult to count with the traditional method. The Milliflex Quantum allows earlier counting, with the microcolonies being smaller and separated.

3.1.4. Limit of Detection. The method was proved to detect $1 \mathrm{CFU}$ of Candida albicans, Aspergillus brasiliensis, Bacillus subtilis, and Escherichia coli (data not shown). The Milliflex Quantum method gave equivalent result to the traditional method (Student's two samples $t$-test, $P \geq 0.05$ ). The Chi2test demonstrated as well the equivalence of results since proportion of growth is similar for the 2 methods (Chi2-test, $P \geq 0.05)$.

3.1.5. Specificity. The specificity of the Milliflex Quantum method was confirmed as all challenged microorganisms were successfully detected during robustness and accuracy tests.

\subsection{Detection of Microorganisms in Pharmaceutical Process} Waters. Different purified waters were sampled in 5 different pharmaceutical plants, at various stages of the pipes. Table 4 summarizes the results obtained at incubation times allowing conforming results with Milliflex Quantum and allowing a stable count with the traditional method.

The contaminants of tested pharmaceutical waters were detected with Milliflex Quantum within a time range of 24 to 40 hours. In comparison, 5 to 7 days were needed to visually count all colonies with naked eyes. The Milliflex Quantum detected the contaminants in waters from 3- to 7-fold faster than the compendial method.

After staining and reincubation, the viability rate conformed to acceptance criteria, proving the nondestructiveness of the Milliflex Quantum method. Identifications using the MicroSEQ platform (Applied Biosystems, Carlsbad, CA, USA) were performed on some colonies stained and reincubated. Common water contaminants as Rhodococcus sp. and Delftia acidovorans and very slow-grower strains as Aquabacterium parvum and Pelomonas saccharophila were identified, proving that the Milliflex Quantum method is fully compatible with identification technology.

\subsection{Specific Case Study: Detection of Slow Grower Microorgan-} isms in Pharmaceutical Process Water. In-process nonsterile pharmaceutical water samples were collected after double reverse osmosis, UV and ozone exposure. Microorganisms being highly stressed in these conditions, an incubation time range from 1 to 7 days was applied before detection with Milliflex Quantum and parallel compendial counting were performed until 21 days.

Figure 2 compares counts obtained with both methods. With the compendial method, all counts increased up to

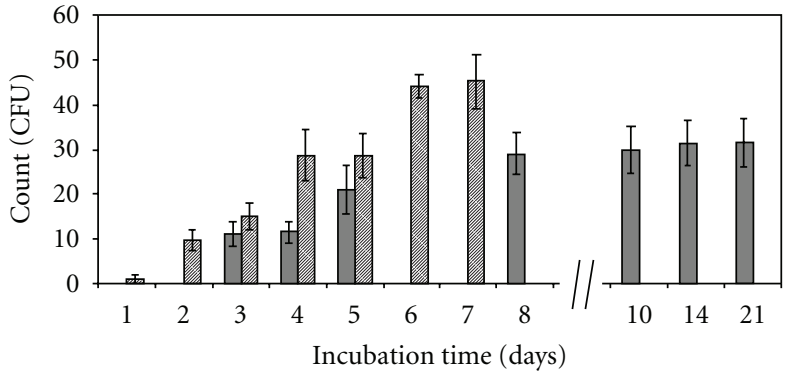

CFU, colony-forming unit

FIgURE 2: Detection of contaminants in pharmaceutical process water: comparison between fluorescence counts obtained with the Milliflex Quantum method (dashed) and counts obtained with the compendial method (solid grey) $(n=5)$. Standard deviation is denoted by the vertical bars.

14 days of incubation and remained stable between 14 and 21 days. The compendial count at 14 days was chosen as the reference for recoveries calculations. The fluorescence recoveries conformed to acceptance criteria from 4 days of incubation, compared to the compendial count obtained after a 14-day incubation (Figures 3(a) and 3(b)). Viability recoveries were calculated comparing CFU count after a 14day incubation on membrane stained with Milliflex Quantum and CFU compendial count at 14 days (Figure 3(a)). Conforming viability detection was achieved on membranes stained after 4 days of incubation. The Milliflex Quantum allowed reducing the time-to-results 3.5 -fold by detecting accurately contaminants after only 4 days. Identifications by sequencing were performed either on reincubated membranes after staining or on compendial membranes. Fourteen genii were identified: for example, Variovorax paradoxus, Afipia broomeae, and Bradyrhizobium japonicum. The latter was the slowest microorganism present in the samples as almost all colonies becoming visible after the 5th day of incubation or reincubation had the macroscopic aspect of Bradyrhizobium japonicum.

\section{Discussion}

We report the validation of the Milliflex Quantum method and the evaluation of its performances for the rapid detection of total microbial contaminants in pharmaceutical water samples. This new rapid method was compared to the traditional membrane filtration method for all tests carried out. The Milliflex Quantum method was demonstrated to be reliable, robust, specific, accurate, and linear over the whole range of assays following these guidelines.

Milliflex Quantum was demonstrated to detect accurately contaminants in 7 pharmaceutical process waters after 24 to 40 hours of incubation, in comparison with 5 to 7 days with the traditional method. An additional sample of highly purified water containing very high stressed microorganisms required 14 days to detect contaminants with the culturebased procedure, whereas Milliflex Quantum enabled to shorten this time-to-result to 4 days. Therefore, Milliflex 


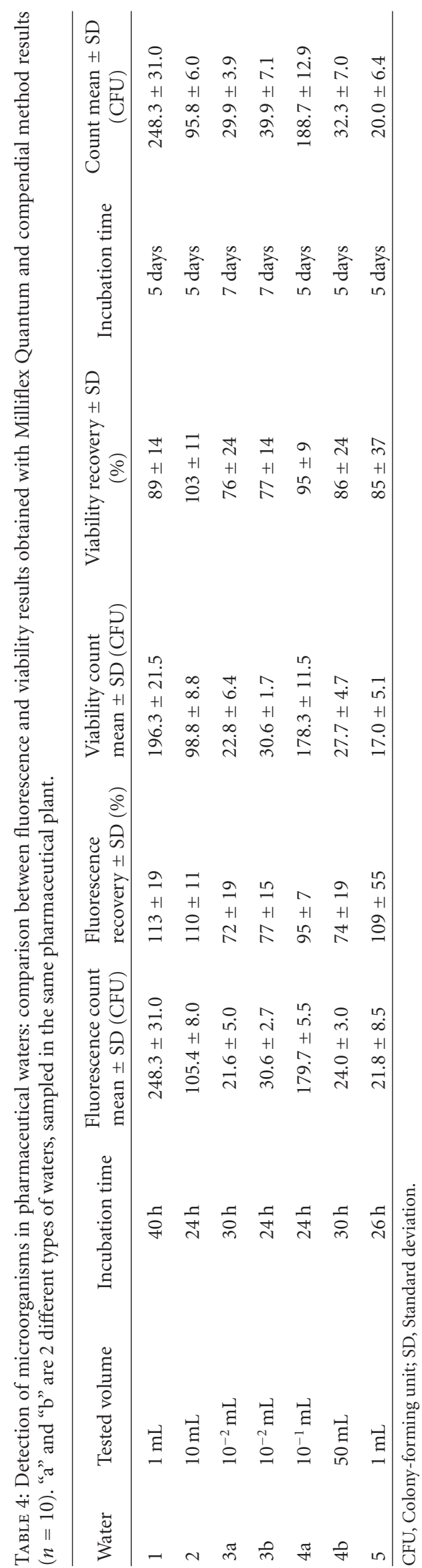




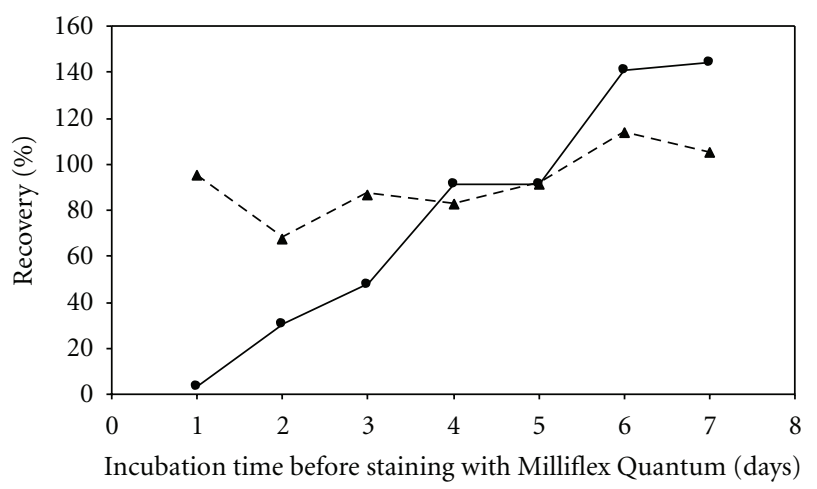

(a)

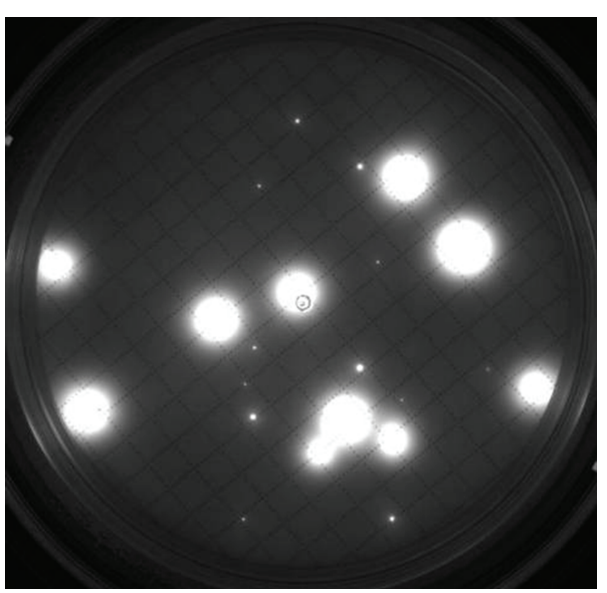

(b)

FIGURE 3: Detection of contaminants in pharmaceutical process water. (a) Fluorescence recovery $(\bullet$, solid line); viability recovery $(\boldsymbol{\Lambda}$, dashed line). Viability recoveries were calculated comparing CFU count after a 14-day incubation with both methods. Each viability recovery is placed on the plot with the incubation time before staining with Milliflex Quantum as abscissa value. (b) Example of picture taken after staining of membrane incubated for 4 days on $\mathrm{R} 2 \mathrm{~A}$ plate at $32.5^{\circ} \mathrm{C}$.

Quantum allows accurate enumeration of contaminants with time-to-results that are 3- to 7-fold shorter than traditional method. Gram-negative and Gram-positive bacteria, as well as yeasts and molds are universally detected with high sensitivity by the alternative method. Some additional tests proved the time-to-results can be shortened if microorganisms are incubated in optimal growing conditions, following microbiological literature instead of Pharmacopeia guidelines. For instance, Pseudomonas aeruginosa and Staphylococcus aureus were accurately detected after 9 hours on TSA at $37^{\circ} \mathrm{C}$, instead of, respectively, 16 hours and 12 hours at the $32.5^{\circ} \mathrm{C}$ temperature recommended by the Pharmacopeia, and Aspergillus brasiliensis needed 17 hours at $37^{\circ} \mathrm{C}$ versus 28 hours at $22.5^{\circ} \mathrm{C}$ (data not shown). Validation of the Milliflex Quantum method, in optimal conditions, as an alternative method for control of microbiological quality is consistent and would enhance time-to-results performances of the Milliflex Quantum system.

The Milliflex Quantum method utilizes mixed cellulose ester membrane filtration for sample preparation, which ensures consistent and reliable results. Large sample volume up to several hundred milliliters can be processed to monitor microbiological quality of very low-contaminated samples. PCR allows testing only small volumes (generally 0.1 to $1 \mathrm{~mL}$ ) after complex sample preparation methods and FCM available systems are limited to the treatment of a $1 \mathrm{~mL}$ volume of sample.

As fluorescent detection is based on metabolism activity of cells, needing integrity of membranes, Milliflex Quantum enables enumeration of all viable-culturable cells, after a short incubation step. Molecular-based methods are unable to distinguish between live and dead cells, which is a shortcoming of methods like PCR $[5,6]$. On the other hand, FCM detection needs to be combined with differential staining techniques to enumerate specifically viable cells.
Viable-culturable cells final result can only be calculated by comparison with the number of plate count, thus requiring the growth and visualization of colonies on traditional agar plate after one or several days incubation [7].

The method was proved to detect $1 \mathrm{CFU}$ while reaching this sensitivity level with molecular-based method requires development of complex and often laborious sample preparation methods [8]. The SPC's claimed sensitivity is $1 \mathrm{CFU}$ with immediate detection but discrimination is often difficult to distinguish between microorganisms and dust and raw results can only be analyzed by high skilled operator after a long specific training. On the other hand, FCM is not adapted to the detection of rare event and enables direct counting of contamination levels down to 100-200 cells per $\mathrm{mL}[9,10]$. Moreover, this technology remains complex to be implemented routinely as data analysis is sophisticated and needs experienced operators $[1,11]$. Therefore, FCM is perfectly suitable to monitoring of drinking water and wastewater treatments, whereas followup of microbiological quality of pharmaceutical or biopharmaceutical waters remains very limited as they may contain very low numbers of viable microorganisms [12].

The staining procedure was demonstrated to be nondestructive since colonies grow after staining and reincubation and can be collected for identification. Sequencing allowed identifying natural contaminants of tested pharmaceutical waters. Moreover, all standard identifications methods (Gram-staining, biochemical tests, PCR, etc.) were applied successfully on other samples after filtration, staining, and reincubation to prove their compatibility with the Quantum procedure (data not shown). The nondestructiveness of the method is an advantage over molecular-based and adenosine triphosphate-based bioluminescence methods, which do not enable to recover and identify contaminants in case of a positive result. 
Implementation and use of new rapid method by industries are limited, among others, by regulatory acceptance $[1,10]$. Milliflex Quantum method is a membrane-filtration and growth-based procedure, very close to the principle of the compendial method. Sample preparation and incubation conditions remain identical to traditional microbiology. These features have the advantage to facilitate validation and reassure regulation agencies for their acceptance of rapid method as routine procedure for water monitoring, replacing longer traditional methods.

Milliflex Quantum was demonstrated to be compatible and efficient in beverages and raw materials matrices (data not shown). The method has been also evaluated for its applicability in the detection of low concentrations of microorganisms in animal cell cultures and cell culture media. Combined to a fast pretreatment method to selectively lyse mammalian cells, the time-to-results were 2-5 times shorter than traditional method [13]. This feature is of great interest for monitoring biotechnology processes including high contaminated cells bioreactors.

We demonstrate in this paper that Milliflex Quantum is a validated and reliable tool enabling the rapid detection and enumeration of microbial contamination in pharmaceutical process waters. This easy-to-use protocol and simple system is totally suitable to be implemented in routine for the monitoring of purified water. The Milliflex Quantum system can be applied to a wide range of filterable products as it was proved to be effective as well with beverages, raw materials, and mammalian cells contaminated matrices.

\section{References}

[1] R. T. Noble and S. B. Weisberg, "A review of technologies for rapid detection of bacteria in recreational waters," Journal of Water and Health, vol. 3, no. 4, pp. 381-392, 2005.

[2] R. Chollet, M. Kukuczka, N. Halter et al., "Rapid detection and enumeration of contaminants by ATP bioluminescence using the milliflex $\AA$ rapid microbiology detection and enumeration system," Journal of Rapid Methods and Automation in Microbiology, vol. 16, no. 3, pp. 256-272, 2008.

[3] L. Yang and R. Bashir, "Electrical/electrochemical impedance for rapid detection of foodborne pathogenic bacteria," Biotechnology Advances, vol. 26, no. 2, pp. 135-150, 2008.

[4] A. Baumstummler, R. Chollet, H. Meder et al., "Development of a nondestructive fluorescence-based enzymatic staining of microcolonies for enumerating bacterial contamination in filterable products," Journal of Applied Microbiology, vol. 110, no. 1, pp. 69-79, 2011.

[5] J. T. Keer and L. Birch, "Molecular methods for the assessment of bacterial viability," Journal of Microbiological Methods, vol. 53, no. 2, pp. 175-183, 2003.

[6] A. Nocker and A. K. Camper, "Novel approaches toward preferential detection of viable cells using nucleic acid amplification techniques," FEMS Microbiology Letters, vol. 291, no. 2, pp. 137-142, 2009.

[7] M. M. T. Khan, B. H. Pyle, and A. K. Camper, "Specific and rapid enumeration of viable but nonculturable and viableculturable gram-negative bacteria by using flow cytometry," Applied and Environmental Microbiology, vol. 76, no. 15, pp. 5088-5096, 2010.
[8] B. S. Byron, Y. Charles, J. Lee-Ann, and M. L. Tortorello, "Sample preparation: the forgotten beginning," Journal of Food Protection, vol. 72, no. 8, pp. 1774-1789, 2009.

[9] F. Hammes, M. Berney, Y. Wang, M. Vital, O. Köster, and T. Egli, "Flow-cytometric total bacterial cell counts as a descriptive microbiological parameter for drinking water treatment processes," Water Research, vol. 42, no. 1-2, pp. 269-277, 2008.

[10] J. Moldenhauer, "Overview of rapid microbiological methods," in Principles of Bacterial Detection: Biosensors, Recognition Receptors and Microsystems, M. Zourob, S. Elwary, and A. Turner, Eds., pp. 49-79, Springer, New York, NY, USA, 2008.

[11] F. Hammes and T. Egli, "Cytometric methods for measuring bacteria in water: advantages, pitfalls and applications," Analytical and Bioanalytical Chemistry, vol. 397, no. 3, pp. 10831095, 2010.

[12] D. T. Reynolds and C. R. Fricker, "Application of laser scanning for the rapid and automated detection of bacteria in water samples," Journal of Applied Microbiology, vol. 86, no. 5, pp. 785-795, 1999.

[13] A. Baumstummler, R. Chollet, H. Meder, C. Rofel, A. Venchiarutti, and S. Ribault, "Detection of microbial contaminants in mammalian cell cultures using a new fluorescencebased staining method," Letters in Applied Microbiology, vol. 51, no. 6, pp. 671-677, 2010. 

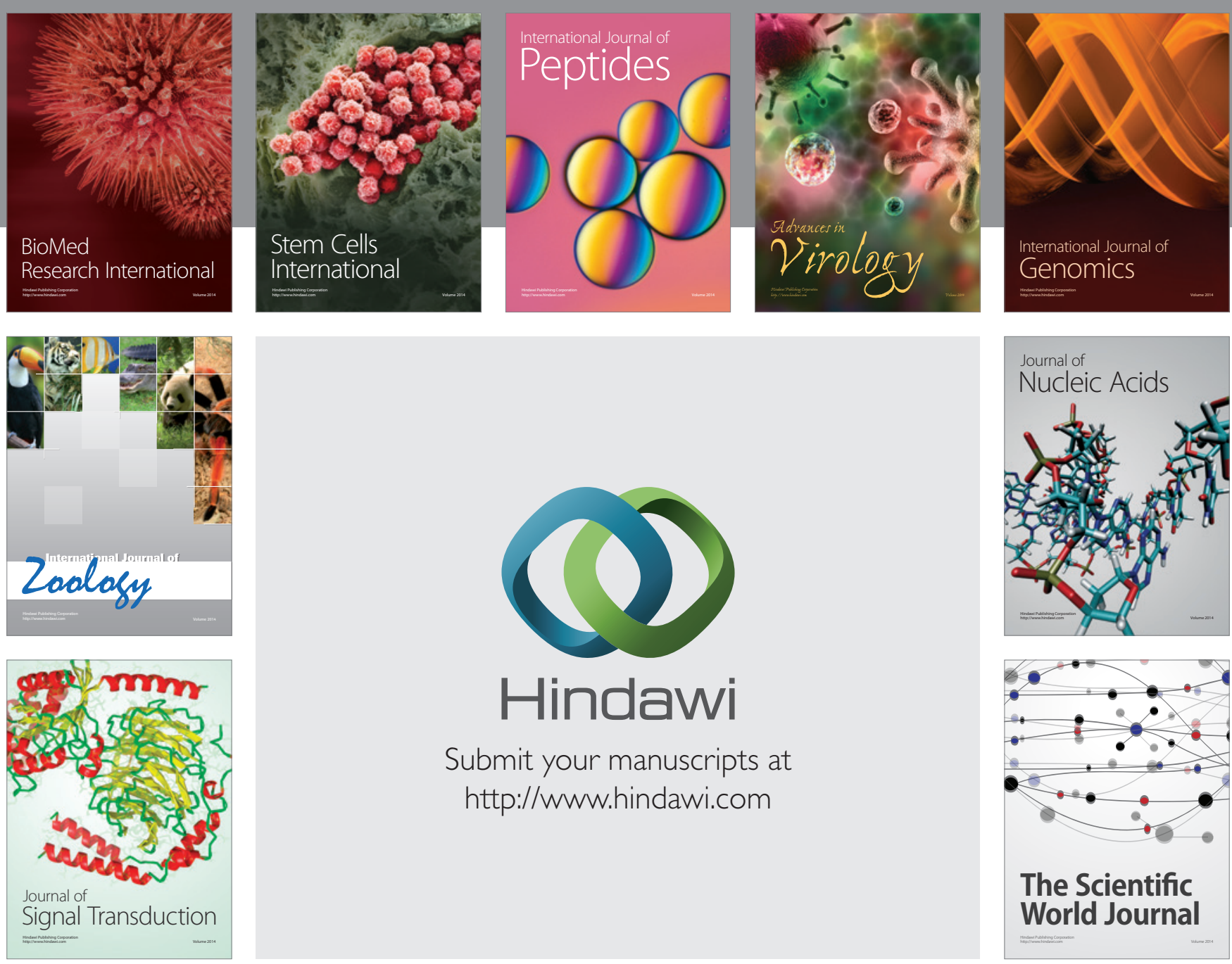

Submit your manuscripts at

http://www.hindawi.com
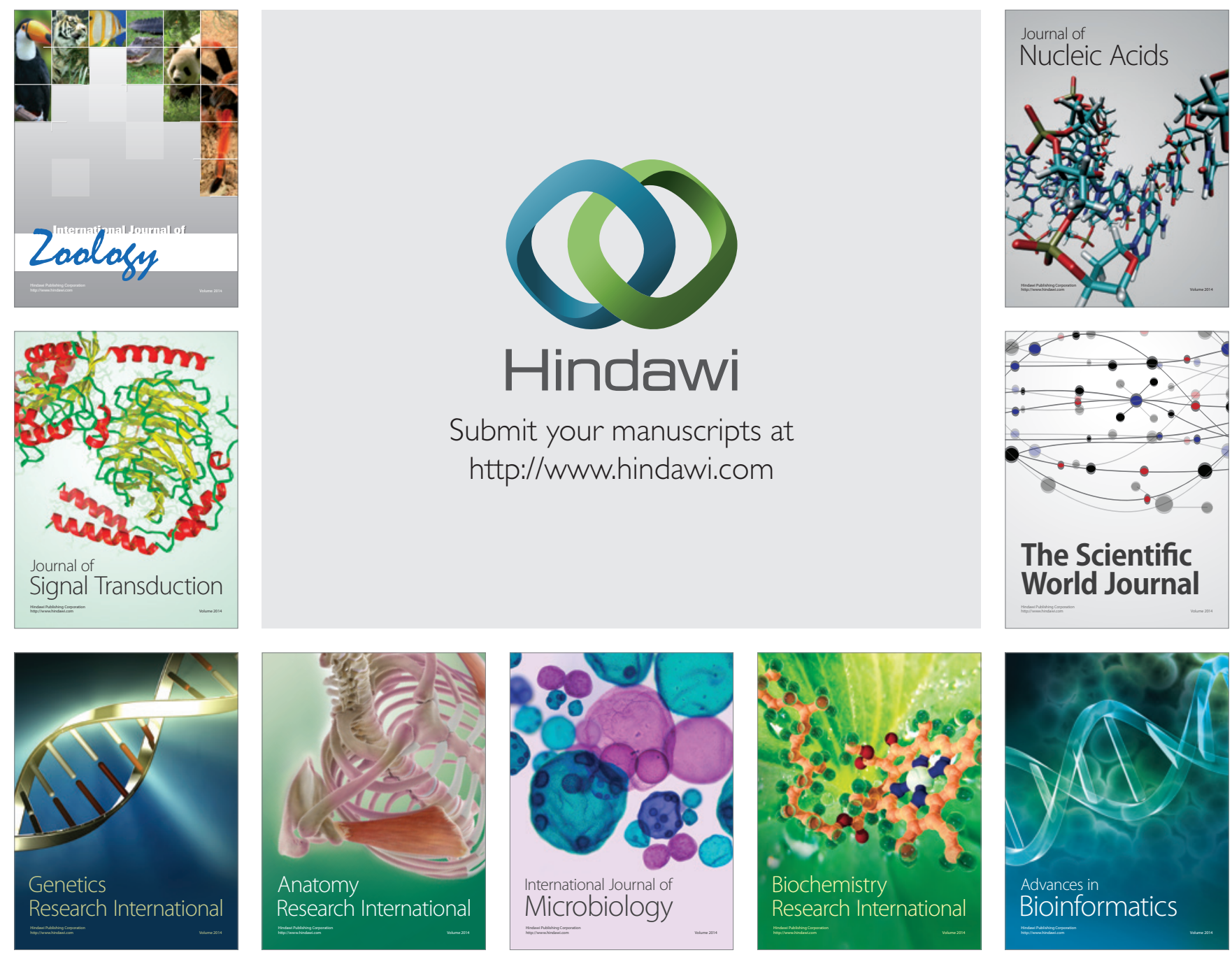

The Scientific World Journal
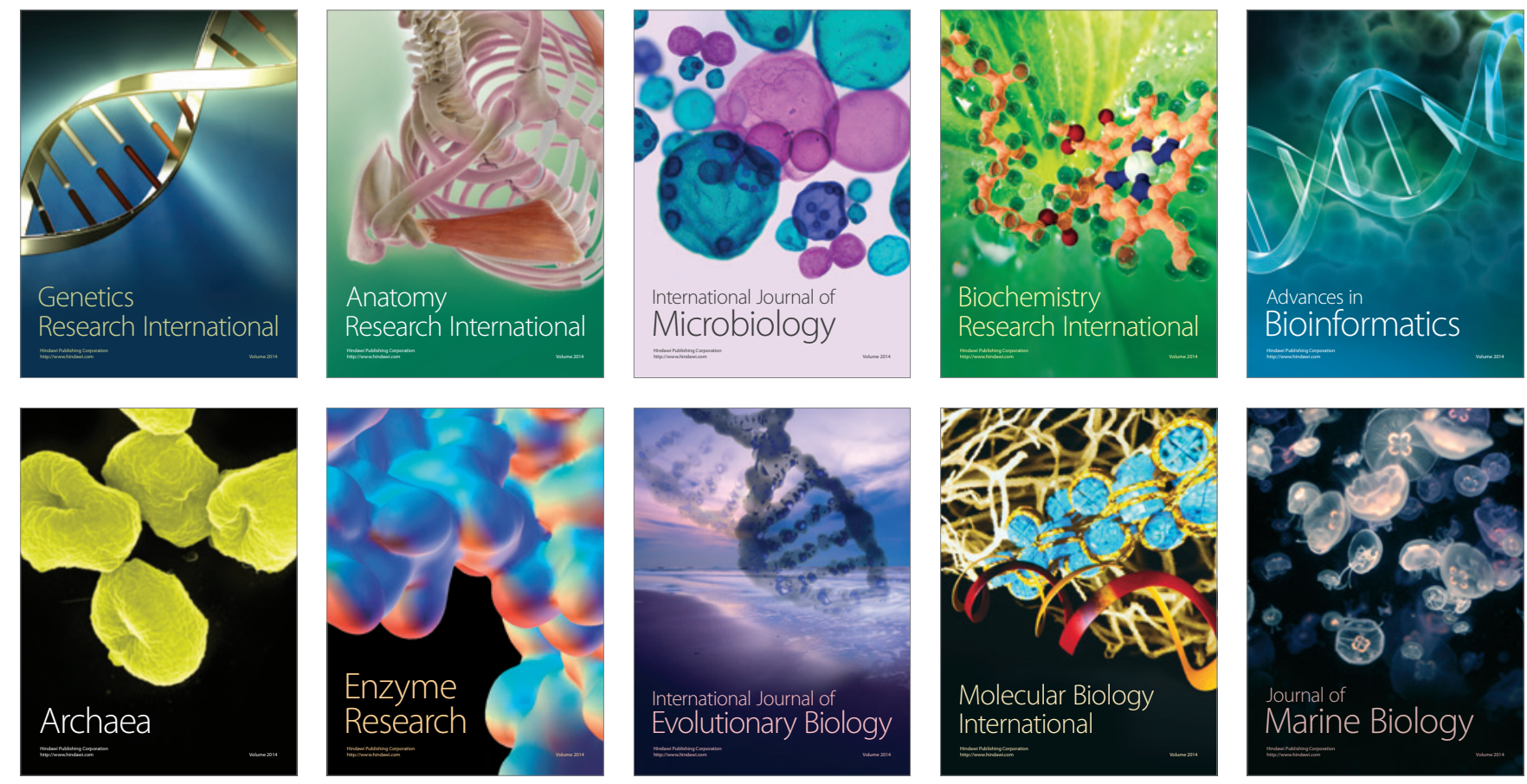